\title{
Multi-vehicle Ad-hoc Network Routing Algorithm with Ant and Quantum Particle Swarm Optimization Combined
}

\author{
Zhiping Wan \\ Xinhua College of Sun Yat-sen University, Guangzhou, 510520, China \\ E-mail:wzp888_0@126.com
}

\begin{abstract}
In order to improve data transfer efficiency of the vehicle self-organizing networks and reduce end transmission delay, this paper presents a Multi-vehicle ad-hoc network routing algorithm with Ant and quantum particle swarm optimization combined (AQVT) . The algorithm based on the thinking of ant colony algorithm that can find the optimal path, through distance relationship between vehicle and roadside access point and vehicle location information to as measures of ant search path, to find the optimal vehicles network routing. and uses the idea of QPSO algorithm that can global search to enhance the global convergence of the algorithm. Experimental results show that, compared MROFDM algorithm and DRIP algorithm, AQVT algorithm can achieve higher data delivery ratio and obtain a lower-end latency.
\end{abstract}

Keywords-Vehicle self-organizing network; more routing algorithm; ant colony; quantum-behaved particle swarm

\section{INTRODUCTION}

With the development of intelligent transportation systems, especially to produce unmanned technology, more and more vehicles start loading electronic equipment to improve road traffic safety. Based on this concept, the vehicle Ad-hoc Network (VANET) that used the vehicle as a signal transmitting and receiving nodes to achieve information exchange between vehicles[1-3], between vehicle and external, which began to be raised and became a hot topic in the field of wireless sensor networks. Feature of VANET is a multi-hop ad hoc networks and high speed mobility, topology changes quickly. Electronic equipment placed on the vehicle can be used to connect to a data server gateway roadside and observational data collection, such as local road conditions, weather, and other observational data traffic information. Vehicle Infrastructure contains some fixed access point (RSUs), it can provide an Internet connection service or traffic systems connection service, so for the vehicle, in order to obtain real-time traffic information, in which the transmission range by a multi-hop access RSUs form is very important[4-5]. Some vehicles can connect by single-hop within its transmission range, and more than a communication range of the vehicle itself is required by multi-hop to communicate.

For the study of VANET, researchers achieved a certain stage of the research results. A movement range oriented forwarding and dynamic multi-copies routing protocol (MROFDM) is proposed in literature[6], with the similarity among the movement models and the local real-time information of vehicles, this protocol sended packets to the movement scope of destination node. At the same time, the copy equilibrium strategy was used to dynamically adjust copy number of different types of packets. MROFDM with some existing multi-copies routing protocols were compare in simulation. A Distributed Real-time Information based Routing Protocol in vehicular ad-hoc networks (DRIP) is proposed in literature[7], Based on the proposed Distributed Real-time Delay Evaluation Scheme (DRES), vehicles obtain the real-time information for the network status of each road, and according to the delay evaluation of each road[8], vehicles use DRIP for an effective data delivery.

\section{ACO AND QUANTUM PARTICLE SWARM OPTIMIZATION}

Ant colony algorithm is a heuristic optimization algorithm proposed by Dorigo M and his colleagues in 1996 . the algorithm inspired by ant colony foraging behavior, which can be used to find the optimal path in the figure. ACO has been applied to many problems[9-10], such as the traveling salesman problem (TSP), scheduling, open shop scheduling, etc. Ant colony algorithm is an effective method to solve the problem of feature selection. The following description of the ant colony algorithm:

The probability $p_{t}(i, j)$ for ant $k$ in time $t$ from node $i$ to node $j$, which may be defined as a state transition rule:

$$
p_{t}(i, j)= \begin{cases}\frac{\tau_{t}(i, j)^{\alpha} \eta_{t}(i, j)^{\beta}}{\sum_{s \in C_{k}} \tau_{t}(i, \mathrm{~s})^{\alpha} \eta_{t}(i, \mathrm{~s})^{\beta}}, \text { if } & s \in C_{k} \\ 0, & \text { else }\end{cases}
$$


Which $C_{k}$ represents a collection of ant $k$ can choose the next hop neighbor nodes, it does not include nodes of the ant $k$ had visited. The parameter $\tau_{t}(i, j)$ is ant $k$ leave pheromone strength in time $t . \eta_{t}(i, j)$ represents a desired ants from node $i$ to node $j, \alpha$ and $\beta$ is a normalization factor, where $\alpha$ is information heuristic factor, $\beta$ decides the importance of the heuristic information. $\eta_{t}(i, j)$ is defined as:

$$
\eta_{t}(i, j)=\frac{1}{d(i, j)}
$$

$d(i, j)$ represents the Euclidean distance between node $i$ and node $j$. After all nodes are traversed by every ant once, the pheromone on the way from $i$ to $j$ can be updated according to the following equation:

$$
\tau_{t}(i, j)=(1-\rho) \tau_{t}(i, j)+\sum_{k=1}^{M} \square \tau_{t}^{k}(i, j)
$$

$\rho$ is pheromone evaporation rate, $M$ is each iteration of the number of ant, $\square \tau_{t}^{k}(i, j)$ is the increment of pheromone from node from $i$ to node $j$. $\square \tau_{t}^{k}(i, j)=\chi / L_{k}, \quad \chi$ is pheromone intensity, $L_{k}$ indicates that the route length.

Quantum behavior of particle swarm optimization algorithm(QPSO) is a global optimization algorithm, the algorithm introduces the average of the best position $C$ that based on PSO algorithm[11-13], To improve the interoperability between groups by waiting effect between particles, and using the form of a probability distribution to make the particles involved in the global search task, which can achieve enhanced global convergence of purpose. In QPSO algorithm, particle swarm moves in accordance with the following formula:

$$
\begin{gathered}
w_{\text {best }}=\frac{1}{N} \sum_{i=1}^{N} P_{i 1}=\left[\frac{1}{N} \sum_{i=1}^{N} P_{i 1}, \cdots, \frac{1}{N} \sum_{i=1}^{N} P_{i m}\right] \\
u_{i d}=\phi P_{i d}+(1-\phi) P_{g d}, \phi=\text { rand } \\
x_{\text {id }}=u_{i d} \pm \lambda \times\left|w_{\text {best }}-x_{i d}\right| \times \ln \left(\frac{1}{V}\right), V=\text { rand }
\end{gathered}
$$

where $P_{i}$ represents the best fitness value of particle $i, x_{i}$ indicating the current position of the particle, $w_{\text {best }}$ is an intermediate position of particles, $u_{i d}$ is random points between $P_{i d}$ and $P_{g d}, \lambda$ is contraction expansion coefficient of QPSO.

\section{AQVT ALGORITHM}

According to the idea of ant colony algorithm, here we set position information of the roadside stationary access points and the vehicle as heuristic information, which relies on the coordinates of roadside access points and the vehicle and the direction of vehicle motion. The spatial relationship of vehicles and roadside access points can be considered for determining the routing of the start time. we define moving vehicles and roadside access point as node, and it can send data packets to detect path. As shown in Figure 1, point A represents the vehicle, $\mathrm{C} 1, \mathrm{C} 2, \mathrm{C} 3, \mathrm{C} 4$ denote the three access point side of the road, respectively. $A \rightarrow B$ indicative of the traveling direction of the vehicle, where the vehicle A through the access point to connect to the server side of the road acquisition information, each roadside access point has a signal transmitting/receiving a fixed radius $R$.

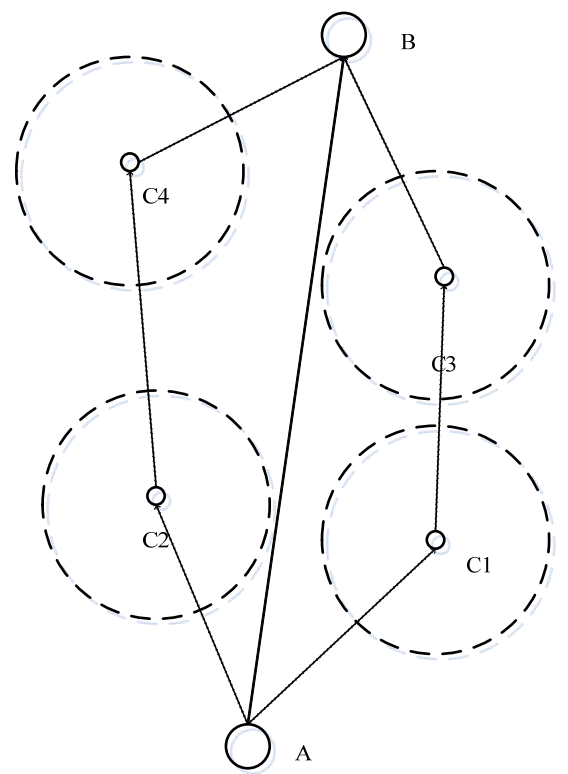

Figure 1 Routing model diagram

According to the idea of the ant colony algorithm, the probability of selecting the first route of the vehicle can be determined communication duration ratio of route, assuming the vehicle through the access points $\mathrm{C} 2$ and $\mathrm{C} 4$, regular expression of the $\phi$ is:

$$
\phi=\frac{4 R}{L_{A C 2}+L_{C 2 C 4}+L_{C 4 B}}
$$

And get a new state transition rules: 
$p_{t}^{k}(i, j)= \begin{cases}\frac{\tau_{t}(i, j)^{\alpha} \eta_{t}(i, \phi)^{\beta}}{\sum_{s \in C_{k}} \tau_{t}(i, s)^{\alpha} \eta_{t, \phi}(i, s)^{\beta}}, \text { if } & s \in C_{k} \\ 0, & \text { else }\end{cases}$

Where $\eta_{t, \phi}(i, \mathrm{~s})$ is the heuristic function, indicating the extent of the heuristic information. $\eta_{t, \phi}(i, \mathrm{~s})$ can be determined by $\phi$ :

$$
\eta_{t, \phi}(i, \mathrm{~s})=\frac{1}{L_{A B} \phi}
$$

Where $L_{A B}$ is Euclidean distance between A and B.

Equation (8) and Equation (9) can determine the probability of ant route selection, however, in order to enhance the global convergence of the algorithm. We have also introduced the idea of QPSO algorithm global search on route selection[14-15], and consider the distance between the vehicle and the first roadside access points, so that the vehicle can quickly access a server publishing or get road information, rather than just the shortest path. Then $\phi$ in the formula (7) can be converted to the expression:

$$
\phi=\frac{4 R}{L_{A C 2}+L_{C 2 C 4}+L_{C 4 B}}+\frac{L_{A C 2}}{L_{A B}}
$$

$t$ is defined as the vehicle to traverse the cycle time, $\square \tau_{t, \phi}{ }^{k}(i, j)$ is pheromone increment in a period of time $t$.

The pheromones of the vehicle travelling on the path can be obtained:

$$
\tau_{t+1, \phi}{ }^{k}(i, j)=(1-\rho) \tau_{t, \phi}{ }^{k}(i, j)+\sum_{k=1}^{M} \square \tau_{t, \phi}{ }^{k}(i, j)
$$

The pheromone increment $\square \tau_{t, \phi}{ }^{k}(i, j)$ can be defined as:

$$
\square \tau_{t, \phi}{ }^{k}(i, j)=\frac{Q}{L_{k}}
$$

\section{THE SIMULATION AND ANALYSIS}

In order to verify the performance of the routing algorithm, we use the NS2 simulator for algorithm simulation[16-17], simulation area test scenarios for $1000 \mathrm{~m}$ $\times 1000 \mathrm{~m}$, roadside access points covering a radius of $150 \mathrm{~m}$, the maximum number of vehicles is 20 , the simulation time is $600 \mathrm{~s}$, each data packet size is 512 bytes[18]. Parameters $\rho=0.5, \quad \alpha=0.6, \quad \beta=0.4, \quad$ group comparison algorithm is MROFDM algorithm that proposed in paper [6] and DRIP algorithm that proposed in paper [7].

In the simulation experiment, we record the algorithm packet delivery success rate under the cases of vehicle speed change, and obtain the results of Figure 2. As can be seen from the figure, with the gradual lifting of the vehicle speed, three algorithms packet delivery success rate gradually decreased, the average packet delivery success rate of AQVT algorithm compared to MROFDM algorithm and DRIP algorithm is increased by $8.6 \%$ and $7.2 \%$, respectively. Therefore, AQVT algorithm can achieve higher data delivery ratio, improve the communication efficiency of the vehicle network.

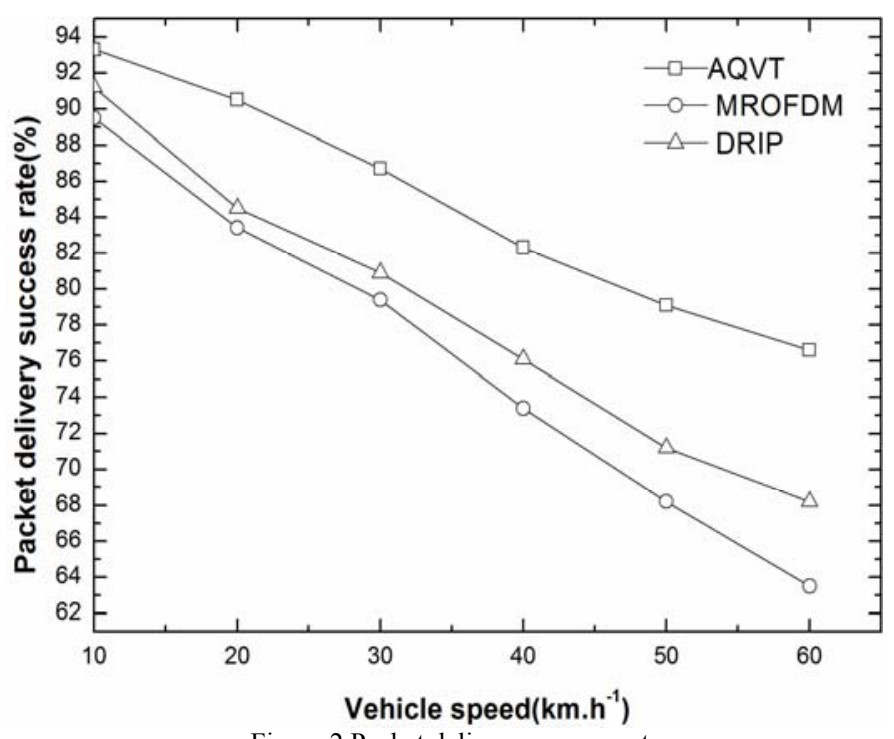

Figure 2 Packet delivery success rate

Figure 3 shows the end to end delay under the case of vehicle speed change for vehicle network. As can be seen from the figure, the faster the vehicle speed, the impact on network latency greater, however, when the vehicle speed increases to a certain extent, delay time variation also gradually reduced. Delay time of DRIP algorithm is maximum, the end to end delay reached $0.154 \mathrm{~s}$ when vehicle speed is $60 \mathrm{~km} / \mathrm{h}$, MROFDM algorithm delays reached $0.142 \mathrm{~s}$, and delay time of AQVT algorithm is minimum, only $0.127 \mathrm{~s}$, thus AQVT algorithm can be more effective to control the network latency.

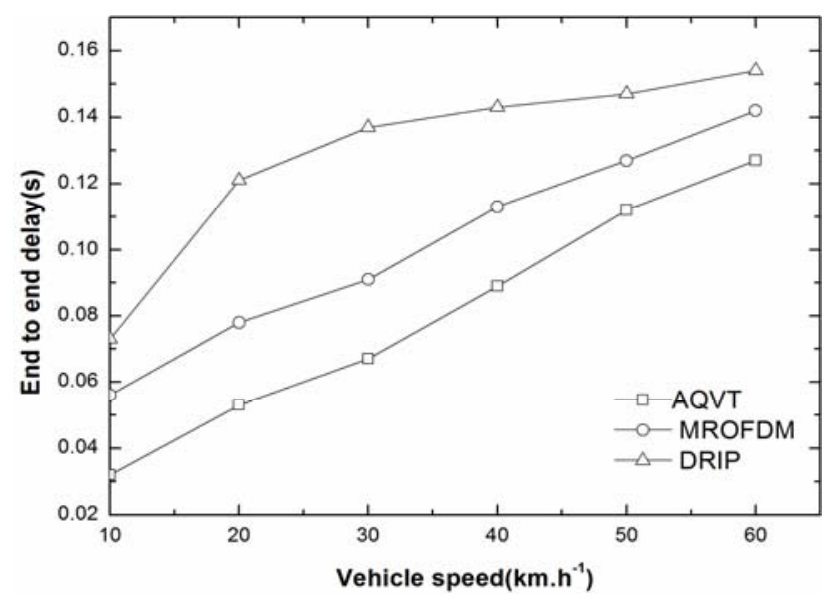

Figure 3 End to end delay 
Figure 4 shows the vehicle network packet loss under vehicle speed change, as can be seen from the figure, the faster the vehicle speed, the greater the packet loss rate, and therefore the vehicle speed has a certain influence on the quality of the communication network of the vehicle. In the whole process of vehicle speed change, the packet loss rate of AQVT algorithm is minimum, packet loss rate of average growth of $2.32 \% / \mathrm{km} \cdot \mathrm{h}^{-1}$. The average growth of packet loss rate of MROFDM algorithm is $3.63 \% / \mathrm{km} \cdot \mathrm{h}^{-1}$, the average growth of packet loss rate of DRIP algorithm is $3.39 \% / \mathrm{km} \cdot \mathrm{h}^{-1}$. When the vehicle speed reaches $60 \mathrm{~km} \cdot \mathrm{h}-1$, the packet loss rate of AQVT algorithms is $12.8 \%$, the packet loss rate of MROFDM algorithms is $21.8 \%$, the packet loss rate of DRIP algorithms is $19.4 \%$. Therefore, the algorithm achieved better results compared to MROFDM algorithm and AQVT algorithm to reduce packet loss rate on the vehicle network.

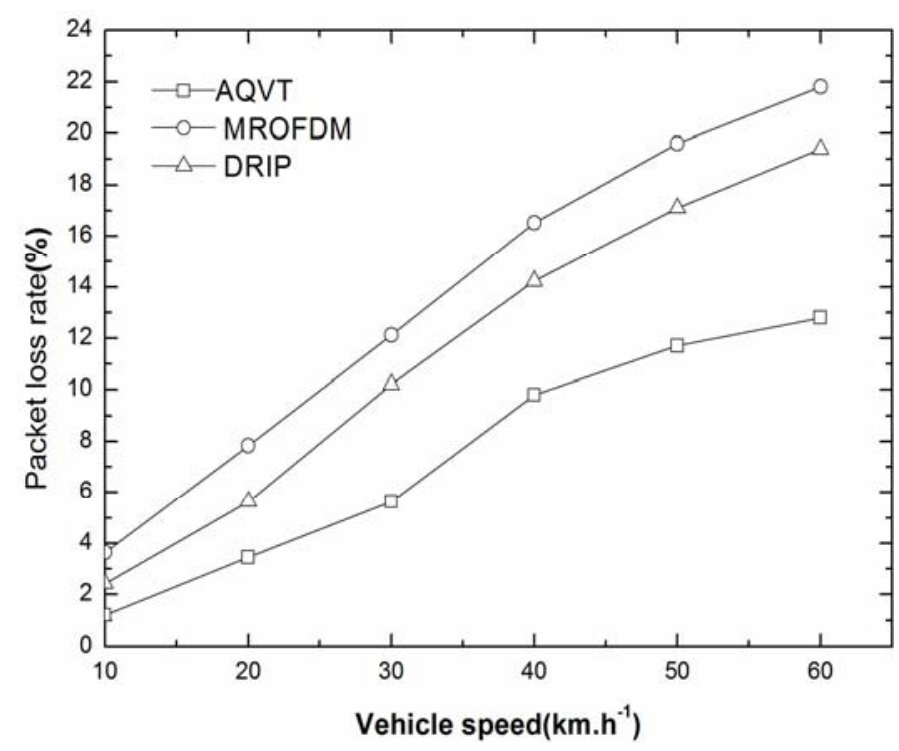

Figure 4 Packet loss rate

\section{CONCLUSIONS}

This paper presents a multi-vehicle ad-hoc network routing algorithm (AQVT) based on ant colony and particle swarm optimization. The algorithm is based on the idea of ant colony algorithm and quantum-behaved particle swarm optimization, through distance relationship between vehicle and roadside access point and vehicle location information to look for the shortest path, at the same time considering the real-time of information exchange between vehicle and road access points, to ensure that the vehicle can get faster traffic information. Experimental results show that the AQVT algorithm has more efficient performance compared MROFDM and DRIP algorithm, especially in packet delivery success ratio and the end to end delay showed better results.

\section{REFERENCES}

[1] Zhang L, Yang G. "An energy - efficient localization algorithm for mobile vehicles in vehicle to vehicle network". Concurr Comp-Pract E. vol. 24, pp. 1213-1222, 2012.

[2] Park I, Sunwoo M. FlexRay network parameter optimization method for automotive applications[J]. Ieee T Ind Electron. vol. 58, pp. 14491459, 2011.

[3] Wan J, Suo H, Yan H, et al. A general test platform for cyberphysical systems: unmanned vehicle with wireless sensor network navigation". Procedia Engineering. vol. 24, pp. 123-127, 2011.

[4] Tejado I, Milanés V, Villagra J, et al. "Fractional network-based control for vehicle speed adaptation via vehicle-to-infrastructure communications". Ieee T Contr Syst T. vol. 24, pp. 780-790, 2013.

[5] Pawlus W, Karimi H R, Robbersmyr K G. "Data-based modeling of vehicle collisions by nonlinear autoregressive model and feedforward neural network". Inform Sciences. vol. 24, pp. 65-79, 2013.

[6] JIANG Hai-tao, ZHANG Hong, LI Qian-mu. "Research on routing protocol of vehicular delay-tolerant networks". Journal on Communications. vol. 24, pp. 76-84, 2013.

[7] SONG Chao, LIU Ming, GONG Hai-Gang. "Distributed Real-Time Information Based Routing Protocol in Vehicular Ad-Hoc Networks". Journal of Software. vol. 24, pp. 466-480, 2011.

[8] Zhu M, Martínez S. "On distributed constrained formation control in operator-vehicle adversarial networks". Automatica. vol. 24, pp. 3571-3582, 2013.

[9] Pan C Z, Lai X Z, Yang S X, et al. "An efficient neural network approach to tracking control of an autonomous surface vehicle with unknown dynamics". Expert Syst Appl. vol. 24, pp. 1629-1635, 2013.

[10] Wang K, Wang H, Xu A. "Research of next generation in-vehicle network FlexRay and its application ". Computer Engineering and Applications. vol. 44, pp. 77-79, 2008.

[11] $M$ orelle K, Lehaire F, Lejeune P. "Spatio-temporal patterns of wildlife-vehicle collisions in a region with a high-density road network". J Nat Conserv. vol. 24, pp. 53-73, 2013.

[12] Zhang F, Buckl C, Knoll A. "Multiple Vehicle Cooperative Localization with Spatial Registration Based on a Probability Hypothesis Density Filter". Sensors. vol. 24, pp. 995-1009, 2014.

[13] Ogren P, Fiorelli E, Leonard N E. "Cooperative control of mobile sensor networks: Adaptive gradient climbing in a distributed environment". Transport Sci. vol. 49, pp. 1292-1302, 2004.

[14] Ekren B Y, Heragu S S, Krishnamurthy A, et al. An approximate solution for semi-open queueing network model of an autonomous vehicle storage and retrieval system". Ieee T Autom Sci Eng. vol. 10, pp. 205-215, 2013.

[15] CHEN Y H, LIU Y H, Qv L D, et al. "Implementation of direct NM logical ring in in-vehicle CAN network". Computer Engineering. vol. 24, pp. 114-116, 2010.

[16] Daniels R C, Heath R W. "Link Adaptation with Position/Motion Information in Vehicle-to-Vehicle Networks". Ieee T Wirel Commun. vol. 11, pp. 505-509, 2012.

[17] Chen J, Zhang L, Kuo Y. "Coverage-enhancing algorithm based on overlap-sense ratio in wireless multimedia sensor networks". Ieee Sens J. vol. 13, pp. 2077-2083, 2013.

[18] Liu Z, Almhana J, McGorman R. Approximating lognormal sum distributions with power lognormal distributions $[\mathrm{J}]$. Ieee $\mathrm{T}$ Veh Technol. vol. 57, pp.2611-2617, 2008. 